\title{
Levitation Force Transition of High-Tc Superconducting Bulks above a Spinning Permanent Magnetic Guideway
}

\author{
$\mathrm{L} \mathrm{Liu}^{1,2}, \mathrm{~J} \mathrm{Li}^{2}$ \\ ${ }^{1}$ Civil Aviation Flight University of China, Guanghan 618307, China \\ ${ }^{2}$ Applied Superconductivity Laboratory, Southwest Jiaotong University, Chengdu 610031, China \\ *Portia.II@163.com
}

Keywords: Superconduct; levitation; magnetic force;

\begin{abstract}
In practical application of a High-Tc Superconducting (HTS) maglev vehicle, the onboard HTS bulks are inevitably exposed to a varying inhomogeneous field due to present processing and assembling technology of the permanent magnetic guideway (PMG). To explore the influence on the levitation forces of $\mathrm{YBa}_{2} \mathrm{Cu}_{3} \mathrm{O}_{7-\mathrm{x}}$ bulk samples in such changing external magnetic field due to different traveling speeds, we experimentally investigated the general transition of said levitation force under such varying external field simulated by a spinning circular PMG. The force waves general attenuated caused by AC loss brought by magnetic field fluctuating. For further information on that levitation dynamic equilibrium, we study experimentally the wave detail and general transition of the dynamic levitation force under different steady velocities between 0 and $120 \mathrm{~km} / \mathrm{h}$. Ultimately the results indicated that the attenuation saturated rapidly in higher speed that would be of benefit to the HTS-PMG system in long-term operating.
\end{abstract}

\section{Introduction}

The onboard YBCO bulks of High-Tc Superconducting (HTS) maglev vehicle experience a changing external magnetic field when traveling along the permanent magnetic guideway (PMG); this changing field depends on the running speed. It can be deduced that these perturbations found in the magnetic field of the PMG can be considered equivalent to an exposure to a complex AC external magnetic field when the bulk is traveling at a speed above the PMG.

AC fields can bring magnetic hysteretic losses [1] and cause irreversible decay of levitation force [2] and trapped field [3], which may cause cyclic force equilibriums affecting the levitation height varying and levitation stability.

For the further engineering application of the HTS Maglev vehicle, the dynamic Maglev properties should be clearly understood. Prior studies on the levitation behavior via AC field perturbations [1], [2] cannot fully imitate the spatial varying applied field under vehicle running performance because it only produced the perturbation along one directional axis. However, with the developed HTS Maglev dynamic measurement system (SCML-03) [4] at the Applied Superconductivity Laboratory (ASCLab) of Southwest Jiaotong University, P. R. China, with a circular permanent magnet guideway (PMG) of $1.5 \mathrm{~m}$ in diameter, the vehicle's translational motion above a PMG can be effectively simulated with the circular PMG allowed to rotate freely underneath [5]. The frequency of the varying external field is proportional to the velocity at which the PMG rotates, and the field strength range is dependent with the working height of the maglev above the PMG.

Consequently, we started to investigate the dynamic behavior of YBCO bulks exposed to such a varying PMG's field at different speeds [6], [7]. This work showed a preliminary understanding on levitation force dynamic equilibrium.

\section{Experimental}

2.1 Experimental Setup and Procedure. As shown in Figure 1, we fixed a sample group of 4 square-shaped HTS bulks to a frame directly connected to four force sensors on the SCML-03 
measurement system for which details are described elsewhere [4]. The specification of the bulks is shown in Table 1, and the samples came from Adelwitz Technologiezentrum GmbH (ATZ), Arzberg, Germany. A container filled with liquid nitrogen (LN2) for cooling down the samples was not connected to the sensor in order to reduce the impact from LN2 volatilization during long time measurements. The data acquisition frequency of the measurements was $1 \mathrm{kHz}$.

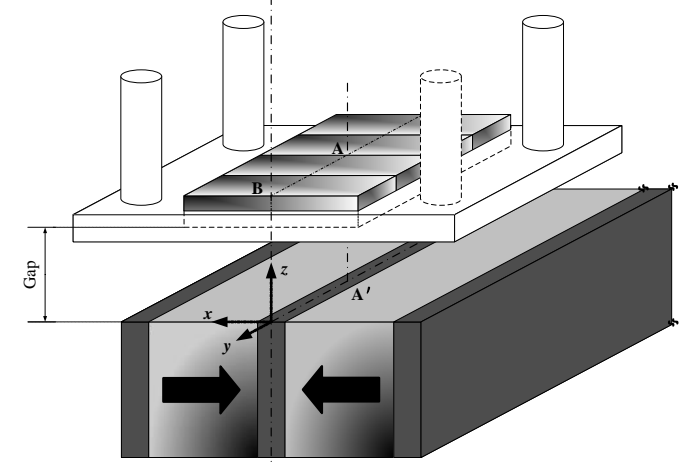

Figure 1. Schematic diagram of the YBCO bulks array above the PMG.

Table 1.Specification of Sample YBCO Bulk

\begin{tabular}{cc}
\hline Parameter & Value \\
\hline Material & YBa2Cu3O7-x \\
Processing & Melt texture; Multiple seeds \\
Technology & $64 \mathrm{~mm} \times 32 \mathrm{~mm} \times 13 \mathrm{~mm}$ \\
Dimension & $0.8-1.1$ Tesla [8] \\
Trapped Field (at 1.4 & \\
T excitation field) & \\
\hline
\end{tabular}

Firstly, the bulks were cooled down in a Zero-Field-Cooling (ZFC) condition. Before the circular PMG was put to spinning for the dynamic levitation force measurement, the YBCO bulks were first kept 10 minutes at the gap of $10 \mathrm{~mm}$ above the PMG circumferential field peak point, corresponding to 0.41 Tesla until the force relaxation reaches a plateau (static force measurement), so the measured dynamic levitation force variations truly reflect the influence of the applied field perturbation. When finished each dynamic measurement case in below section, samples were heated and cooled down again.

2.2 Dynamic Force Measurement. It is considered the levitation force attenuation is closely related to the varying of applied field which depends on the rotational velocity of the circular PMG. Four cases were carried out for the investigation of the force transition of the HTS samples with the following varying applied field as follow cases:

The rotational PMG was accelerated from $0 \mathrm{~km} / \mathrm{h}$ to $1.45 \mathrm{~km} / \mathrm{h}$. The bulks array were kept at a gap of $15 \mathrm{~mm}$. The measurements at steady velocity would last for about 5 minutes.

The rotational PMG was accelerated from $0 \mathrm{~km} / \mathrm{h}$ to $30 \mathrm{~km} / \mathrm{h}$ and then decelerated back to $0 \mathrm{~km} / \mathrm{h}$ step by step at a rate of $7.44 \mathrm{~km} / \mathrm{h}$ each step. The measurements at steady velocity would last for about 5 minutes and the measurements were carried out at a gap of $10 \mathrm{~mm}$, and the follows are the same configuration.

The rotational PMG was accelerated from $0 \mathrm{~km} / \mathrm{h}$ to $60 \mathrm{~km} / \mathrm{h}$ and then decelerated back to $0 \mathrm{~km} / \mathrm{h}$ step by step at a rate of $7.44 \mathrm{~km} / \mathrm{h}$ each step.

The rotational PMG was accelerated from $0 \mathrm{~km} / \mathrm{h}$ to $119 \mathrm{~km} / \mathrm{h}$ and then decelerated back to $0 \mathrm{~km} / \mathrm{h}$ step by step at a rate of $29.75 \mathrm{~km} / \mathrm{h}$ each step.

After each rotation ending (velocity returned to $0 \mathrm{~km} / \mathrm{h}$ ), there was an interval so static measurements can be performed. 


\section{Results and discussion}

3.1 3.1. Magnetic field and levitation force oscillation. The field peak area was nearby central axis in the cross-section of the PMG as in Figure 1, and the circumferential field profiles at that central axis around the circular PMG was shown in Figure 2. Small gap meant preferable vertical magnetic flux density $\mathrm{Bz}$ which might cause to a preferable levitation force. The negative peak of $\mathrm{Bz}$ at a gap of 15 $\mathrm{mm}$ was $0.27 \mathrm{~T}$, and the field oscillated between $0.27 \mathrm{~T}$ and $0.3 \mathrm{~T}$ along the circumferential phase as shown in Figure 3. And the measured force sensitively oscillated with the applied field perturbation between positive peaks and negative peaks as in Figure 3. The profile in Figure 3 and Figure 4 were very similar due to the force was caused by the interaction between the induced current and applied field. The force oscillation could affect levitation performance and cause vibrations [7] of the free levitation body, but we focus on the general tendencies of the levitation force behavior in this paper.

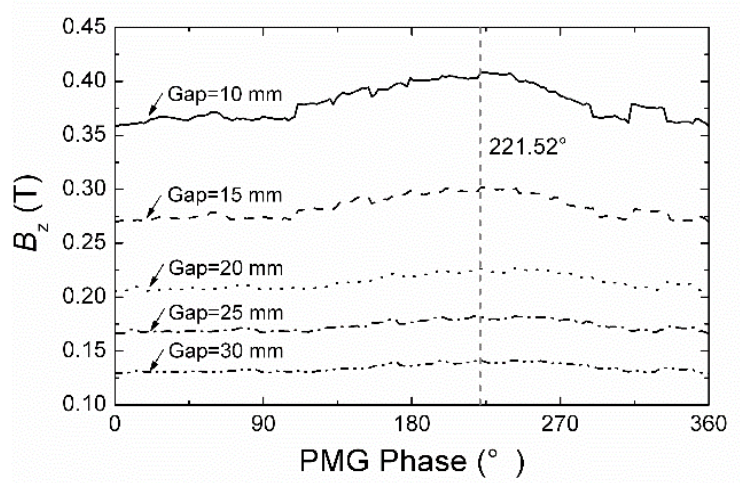

Figure 2. Circumferential vertical magnetic flux density profile along the circle of central axis of the PMG. Bz was between $0.27 \mathrm{~T}$ and $0.3 \mathrm{~T}$ at a gap of $15 \mathrm{~mm}$, while between $0.36 \mathrm{~T}$ and $0.41 \mathrm{~T}$ at a gap of $10 \mathrm{~mm}$.

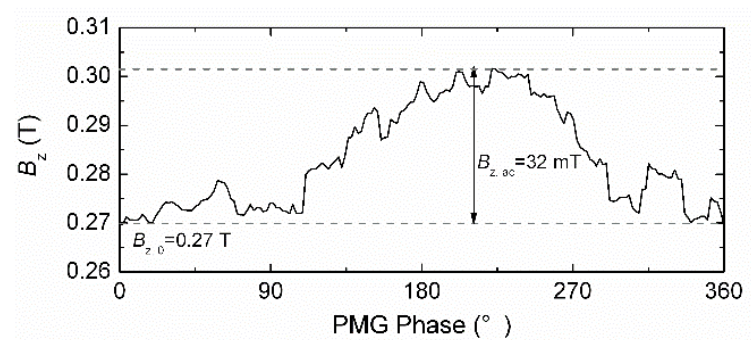

Figure 3. Bz profile at a gap of $15 \mathrm{~mm}$. The magnetic field oscillated at a scale of $32 \mathrm{mT}$, which might cause force Oscillation.

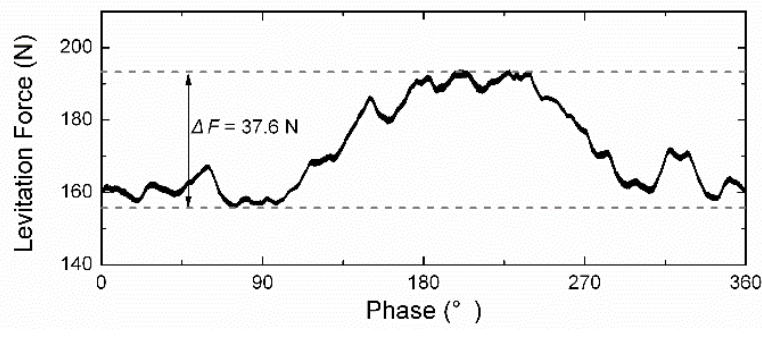

Figure 4. Oscillation of the levitation force after the PMG was put to spinning at $1.45 \mathrm{~km} / \mathrm{h}$ closely corresponded to $\mathrm{Bz}$ profile.

$3.20 \mathrm{~km} / \mathbf{h}$ to $30 \mathrm{~km} / \mathrm{h}$ The. The levitation force transition shows three main phases in Figure 5: one static phase followed by a dynamic phase where the rotational velocity goes from zero to 7.44 $\mathrm{km} / \mathrm{h}$ for 300 seconds then to $15 \mathrm{~km} / \mathrm{h}$ for 300 seconds then to $22 \mathrm{~km} / \mathrm{h}$ for 300 seconds then $30 \mathrm{~km} / \mathrm{h}$ for 300 seconds, back to $0 \mathrm{~km} / \mathrm{h}$ as a reversed step by step and a third phase where the levitation force is measured for another 600 seconds. In the initial static phase, the samples were firstly left to relax for 600 seconds in the static mode given that the levitation force has a decay when it was firstly exposed to a permanent magnet [9] so that the effect of the rotating PMG on the levitation force is reflected more accurately in our data.

The scale between positive peaks and negative peaks in Figure 6 corresponded to the enveloped grey area in Figure 5. The reason was that the waves were too close to hardly clarified with $1 \mathrm{~Hz}$ data acquisition frequency. In Figure 6, it was clearly seen that the force was also Oscillating back and forth approximately from $275 \mathrm{~N}$ to $220 \mathrm{~N}$. The grey measured force rang between positive peaks and negative peaks shifted slightly at different velocities, which increased with the increasing velocities while decreased with the decreasing velocities due to drastic vibration of the spinning PMG. In order to discuss the general tendencies of the levitation force behaviour, we calculated an average force as the Average line shown in Figure 5. 
The average values did not shift at different velocities (Figure 5) but rather it follows a decaying pattern as long as the PMG keeps spinning. The AC components of applied field generated by the PMG spinning periodically penetrates in and out of the sample edge affects the induced currents distribution and the range of the penetration depth, which causes force oscillating and hysteresis losses due to flux movement. The heat generated by hysteresis loss decreases the local shielding current so that field penetrates deeper into the sample and the levitation force attenuates. Until a new thermal equilibrium is established, the force variation does not reach a plateau to stabilize. Therefore, the deceleration phase from $30 \mathrm{~km} / \mathrm{h}$ to zero could not stop the attenuation.
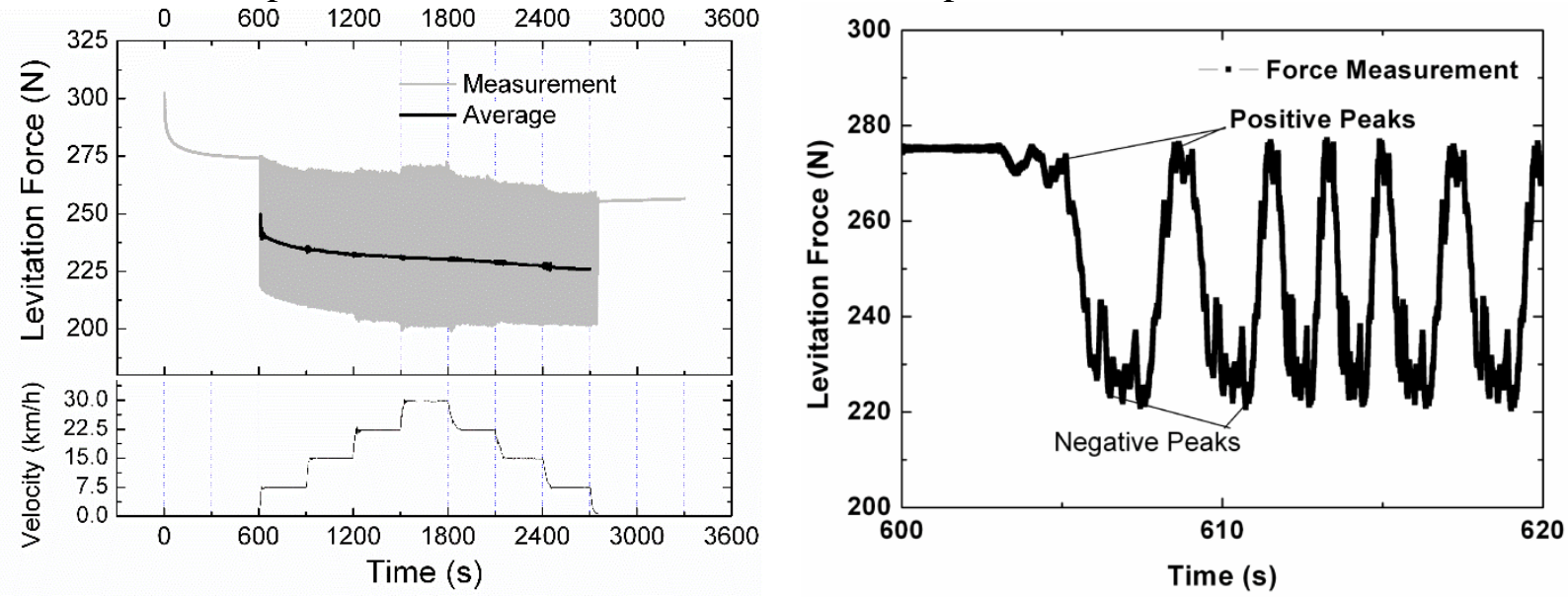

Figure 5. Levitation force and rotational linear velocity as a function of time in experiment Case 2: $0 \rightarrow 30 \mathrm{~km} / \mathrm{h}$.

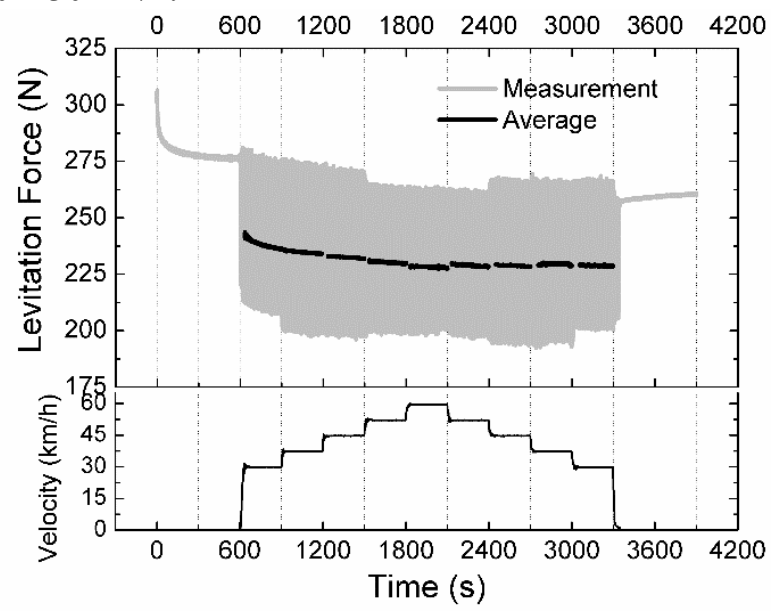

Figure 6. Oscillation of the levitation force after the PMG was put to spinning at a velocity of 7.44 $\mathrm{km} / \mathrm{h}$.

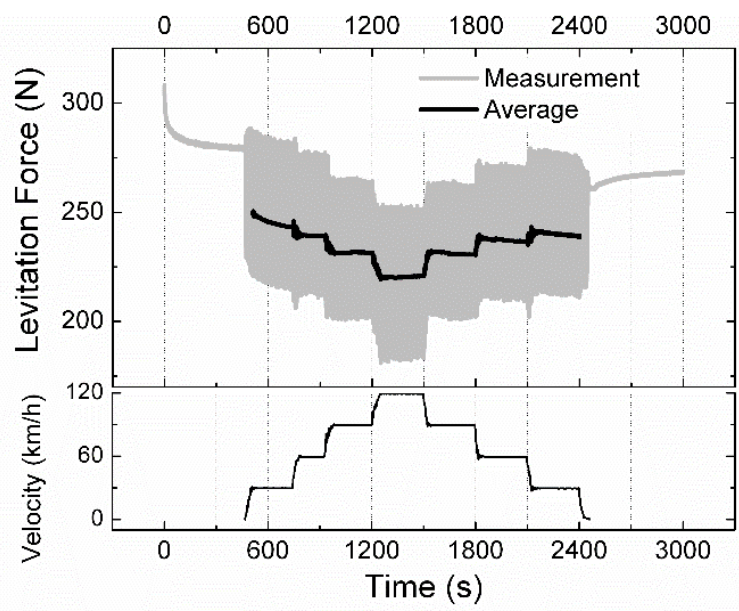

Figure 7. Levitation force and rotational linear velocity as a function of time in experiment Case 3 : $0 \rightarrow 60 \mathrm{~km} / \mathrm{h}$.

Figure 8. Levitation force and rotational linear velocity as a function of time in experiment Case 4: $0 \rightarrow 119 \mathrm{~km} / \mathrm{h}$.

$3.30 \mathrm{~km} / \mathrm{h}$ to $60 \mathrm{~km} / \mathrm{h}$. The measured levitation force curve in Figure 7 also shifted with different velocities, while the average curve was a little stagger. The reason for that stagger might be that the centrifugal force cause a slight deformation for field reduction of the PMG at a high rotating velocity. However, the force attenuating tendency was still absolutely observed.

$3.40 \mathrm{~km} / \mathrm{h}$ to $119 \mathrm{~km} / \mathrm{h}$. The levitation force curves in Figure 8 showed an apparently sharp decay or recovery stage while speed shifting, that because the field varied with the deformation of the permanent magnetic guideway. Therefore, it was hardly to divide whether the force was attenuating or not, but the static force measurement after the dynamic was obviously smaller than the former relaxed force at time of 450 second. 


\section{Conclusions}

We experimentally investigated the behavioral characteristics of the levitation force of a set of superconductors suspended above a spinning PMG at different rotational velocities. At a low rotational velocity, the levitation force keeps attenuating independent of the velocity variation. At high velocities, the levitation force abruptly decreases when there is a change in speed and recovers to almost the original value after going back to the previous lower steady speed value. Repetitive acceleration and deceleration affected the recovery of the levitation force only slightly that made us have the reason to believe that relate to the deformation of the permanent magnetic guideway.

Consequently, we came to a preliminary conclusion that the general transition of levitation force trends to reach a dynamic equilibrium in a steady speed especially at high speeds, while the energy loss dominates in force decay at low speeds. More theoretical work is needed on the levitation performance estimation as well as experimental work at low speeds and speeds higher than $120 \mathrm{~km} / \mathrm{h}$ for future HTS vehicle applications.

\section{References}

[1] L.C. Zhang, J.S Wang, S.Y. Wang, Q.Y. He. 2007. Physica C, 459: 43-44.

[2] M. X. Liu, S.Y.Wang, J. S. Wang,G. T. 2009. J. Low Temp. Phys, 155: 169-176.

[3] H. Ueda, M. Itoh, A. Ishiyama. 2003. IEEE Trans. Appl. Supercond, 13(2): 2283-2286.

[4] J. S. Wang, S. Y. Wang, C. Y. Deng, Y. W. Zeng, L. C. Zhang, Z. G. Deng, J. Zheng, L. Liu, Y. Y. Lu, Minxian Liu, Y. H. Lu, Y. G. Huang, Y. Zhang. 2008. IEEE Trans. Appl. Supercond, 18(2): 791-794.

[5] L. Liu, J.S. Wang, Z.G. Deng, S.Y. Wang, J. Zheng, J. Li. 2010. J Supercond. Nov. Magn, 23(5): 597-599.

[6] Z. G. Deng. 2009. PhD. Thesis. Experimental researches on the dynamic levitation characteristics of high temperature superconductor YBCO bulks under moving applied magnetic field, Southwest Jiaotong Univ. Chengdu China.

[7] J. Zheng, Z. G. Deng, L. L. Wang, L. Liu, Y. Zhang, S. Y. Wang, J. S. Wang. 2007. IEEE Trans. Appl. Supercond, 17(2): 2103-2016.

[8] U. Floegel-Delor, R. Rothfeld, D. Wippich, B. Goebel, T. Riedel, F. N. Werfel. 2007. IEEE Trans. Appl. Supercond, 17(2): 2142-2145.

[9] Y. Yeshurun, A. P. Malozemoff, A. Shaulov. 1996. Rev. Mod. Phys, 68(3): 911-949.

[10] J. Ogawa, S. Fukui, T. Oka, M. Yamaguchi, T. Sato, M. Takada, R. Sato, M. Tomita. 2007. IEEE Trans. Appl. Supercond, 17(2): 3024.

[11] Y. Zushi, I. Asaba, J. Ogawa, K. Yamagishi, O. Tsukamoto. 2005. Cryogenics, 45: 17-22.

[12] M. Wilson. 1983. Superconducting Magnet (Oxford: Oxford Science Publisher)

[13] H. Shimizu, H. Ueda, M. Tsuda, A. Ishiyama. 2002. IEEE Trans. Appl. Supercond, 12(1): 820-823. 